\title{
Sound radiation from exponentially growing and decaying surface waves
}

\author{
N.D. Sandham ${ }^{\mathrm{a}, *}$, C.L. Morfey ${ }^{\mathrm{b}}$, Z.W. Hu ${ }^{\mathrm{a}}$ \\ a Aeronautics and Astronautics, School of Engineering Sciences, University of Southampton, Southampton SO17 1BJ, UK \\ ${ }^{\mathrm{b}} I S V R$, University of Southampton, Southampton SO17 1BJ, UK
}

Received 13 May 2005; received in revised form 20 September 2005; accepted 19 October 2005

Available online 13 December 2005

\begin{abstract}
A simplified model problem is used to illustrate some of the parameters controlling the radiation of sound into an ambient medium due to the growth and subsequent decay of subsonic travelling waves, such as may occur via non-linear interactions in turbulent free shear flows. It is shown that substantial sound may be generated by apparently subsonic modes as a result of their growth and decay characteristics. Low frequency modes that saturate over a short timescale are the most effective radiators.
\end{abstract}

(C) 2005 Elsevier Ltd. All rights reserved.

\section{Introduction}

Mechanisms of noise generation are relatively less well understood for subsonic jets than for supersonic jets [1] where turbulence-driven linear instability modes of the jet column act as important sound sources. In subsonic jets the radiation efficiency of such modes is low and it appears likely that non-linear interactions play a role, either as interactions between primary instability waves or (as in the original Lighthill [2] analysis) as interactions between fully-turbulent velocity components. In Section 2, we consider an acoustic model problem related to the first possibility, namely non-linear interaction between two instability modes of the jet column, as a first step towards showing how such an interaction can lead to enhanced sound radiation compared with that emitted directly by each mode according to linear theory.

The model problem is that of sound radiation from an infinite plane boundary, generalised to allow for temporal growth and decay of travelling waves on the boundary. The well-known solution for compressible flow past a wavy wall (see e.g. Ref. [3]) shows that Mach waves are only radiated when the relative Mach number between the wall and the free stream is greater than one. Likewise when a stationary medium is excited by a vibrating plane boundary [4], sound is only radiated into the far field from supersonicallytravelling components of the wall motion. By contrast, when in the latter case the surface wave amplitude grows and decays along the wall it is possible to have sound radiation even when the relative Mach number of the travelling-wave zero crossings is subsonic. In their model of jet noise radiation from instability modes,

*Corresponding author. Tel.: + 442380594872 ; fax: + 442380593058.

E-mail address: n.sandham@soton.ac.uk (N.D. Sandham). 
Crighton and Huerre [5] explored a limited part of the parameter space of this problem, in which the wave packet envelope variation is asymptotically slow compared to the primary wavenumber. Here we consider solutions to a related temporal problem involving forced waves on a plane boundary; no restriction is placed on the growth rate, and the aim is to show how the various parameters control the sound radiation.

\section{Model problem}

The geometry of the problem is shown in Fig. 1. A uniform fluid at rest occupies the region $y>0$, and is excited by small-amplitude normal vibration of the boundary at $y=0$. As a starting point we take the $(x, z)$ Fourier transformed wave equation in velocity potential $\phi$ given by

$$
\frac{1}{c^{2}} \frac{\partial^{2} \phi}{\partial t^{2}}+\left(k_{x}^{2}+k_{z}^{2}\right) \phi=\frac{\partial^{2} \phi}{\partial y^{2}},
$$

where wavenumbers in the $x$ and $z$ coordinates are denoted $k_{x}$ and $k_{z}$, respectively, and $c$ denotes the constant sound speed. This wave equation is solved in the domain $y \geqslant 0$, subject to the boundary condition at $y=0$ given by

$$
\left(\frac{\partial \phi}{\partial y}\right)_{0}=A(t) \exp (-\mathrm{i} \omega t+\mathrm{i} \psi)
$$

where $\omega$ is a (real) frequency and $\psi$ is a phase angle. The amplitude function $A$ satisfies

$$
\frac{\mathrm{d} \ln A}{\mathrm{~d} t}=\sigma,
$$

with the local growth rate $\sigma$ specified as

$$
\begin{aligned}
\sigma & =\sigma_{0} \quad \text { for } t<-\Delta \\
& =-\frac{\sigma_{0} t}{\Delta} \quad \text { for }-\Delta \leqslant t \leqslant \Delta \\
& =-\sigma_{0} \text { for } t>\Delta,
\end{aligned}
$$

where $\sigma_{0}$ and $\Delta$ are positive constants. By integration of Eq. (3), taking the amplitude equal to one at $t=0$ we have the amplitude history given by

$$
\begin{aligned}
\ln A & =\frac{\sigma_{0} \Delta}{2}+\sigma_{0} t \quad \text { for } t<-\Delta \\
& =-\frac{\sigma_{0} t^{2}}{2 \Delta} \quad \text { for }-\Delta \leqslant t \leqslant \Delta \\
& =\frac{\sigma_{0} \Delta}{2}-\sigma_{0} t \text { for } t>\Delta .
\end{aligned}
$$

This specifies a problem in which spatially-harmonic waves with wavenumber $\left(k_{x}, k_{z}\right)$ parallel to the boundary grow exponentially with time at early (negative) times, saturate over a controlled timescale, and then decay

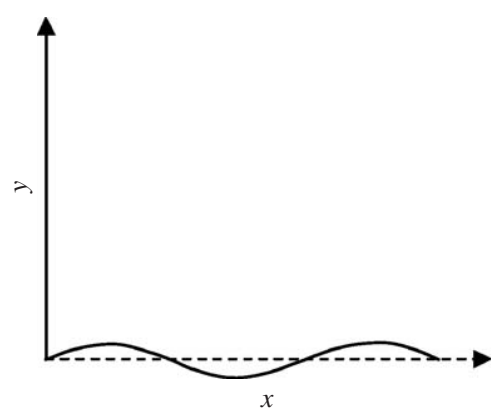

Fig. 1. Schematic of the solution domain. A spatially-sinusoidal normal velocity is applied over the entire plane $y=0$. 

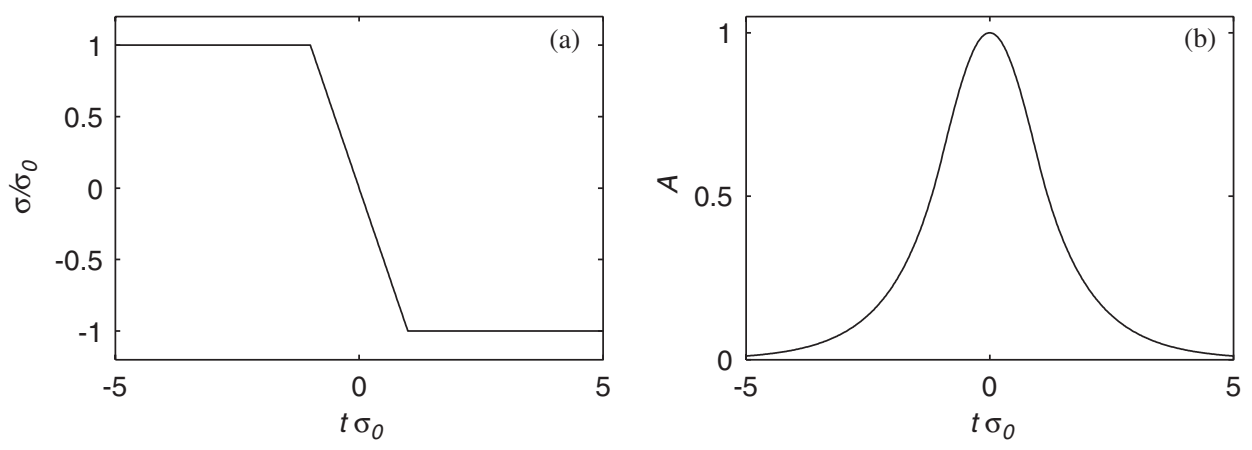

Fig. 2. Schematic of the input forcing: (a) growth rate variation from Eq. (3), (b) the resulting amplitude variation with time.

exponentially for large (positive) times (see Fig. 2 for a schematic of the behaviour). This problem is less restricted than the Crighton and Huerre [5] problem, in which the wave packet envelope is assumed to vary spatially over a much longer scale than the Fourier wave. Our interest is in the extent to which the controlling parameters (wavenumber, sound speed, frequency and saturation timescale, normalised versions of which are defined in the next section) are active in cutting off the radiation from subsonic modes, where 'subsonic' in the context of the specified model parameters means $|k|>\omega / c$, with $k^{2}=k_{x}^{2}+k_{z}^{2}$.

It is instructive to consider Fourier components of the amplitude variation in Eq. (5). Due to the amplitude variation with time there will be contributions at all frequencies, including those corresponding to supersonic phase speeds. The possibility of sound radiation from 'subsonic' forcing, as defined above, is therefore consistent with sound radiation from that portion of the forcing spectrum that is supersonic in a conventional wavy-wall sense.

\subsection{Zero wavenumber analytical solution for a step change in growth rate $(\Delta \rightarrow 0)$}

During the exponential growth phase $t<-\Delta$ the solution to Eqs. (1) and (2) is

$$
\phi=-\frac{\exp \left(-\gamma y+\left(\sigma_{0}-\mathrm{i} \omega\right) t+\mathrm{i} \psi\right)}{\gamma},
$$

where

$$
\gamma= \pm \sqrt{k_{x}^{2}+k_{z}^{2}+\frac{\left(\sigma_{0}-\mathrm{i} \omega\right)^{2}}{c^{2}}}
$$

The sign of the square root is dictated by causality: the real part of $\gamma$ must be positive to avoid solutions which grow exponentially with $y$. In the limit $\sigma_{0} / \omega \rightarrow 0$, corresponding to the time-harmonic problem of Ref. [4], there is a sharp cutoff in sound radiation as the phase speed $\omega /|k|$ along the boundary changes from supersonic to subsonic. For finite $\sigma_{0}$ this transition is smoothed out and the sound field decays exponentially with distance $y$ from the boundary for all phase speeds. At the same time it grows exponentially with time, up to $t=-\Delta$.

For $k_{x}=k_{z}=0$ and $\Delta \rightarrow 0$ (i.e. a sudden change in growth rate from $\sigma=\sigma_{0}$ to $\sigma=-\sigma_{0}$ ) the solution for $t>0$ is found in two parts. For $y \geqslant c t$ we have

$$
\phi=-\frac{c \exp \left(-\left(\sigma_{0}-\mathrm{i} \omega\right)(y / c-t)+\mathrm{i} \psi\right)}{\sigma_{0}-\mathrm{i} \omega},
$$

which is a wave travelling outwards in $y$. For $y<c t$, we have

$$
\phi=\frac{c \exp \left(\left(\sigma_{0}+\mathrm{i} \omega\right)(y / c-t)+\mathrm{i} \psi\right)}{\sigma_{0}+\mathrm{i} \omega}-c \exp (\mathrm{i} \psi)\left(\frac{1}{\sigma_{0}-\mathrm{i} \omega}+\frac{1}{\sigma_{0}+\mathrm{i} \omega}\right),
$$




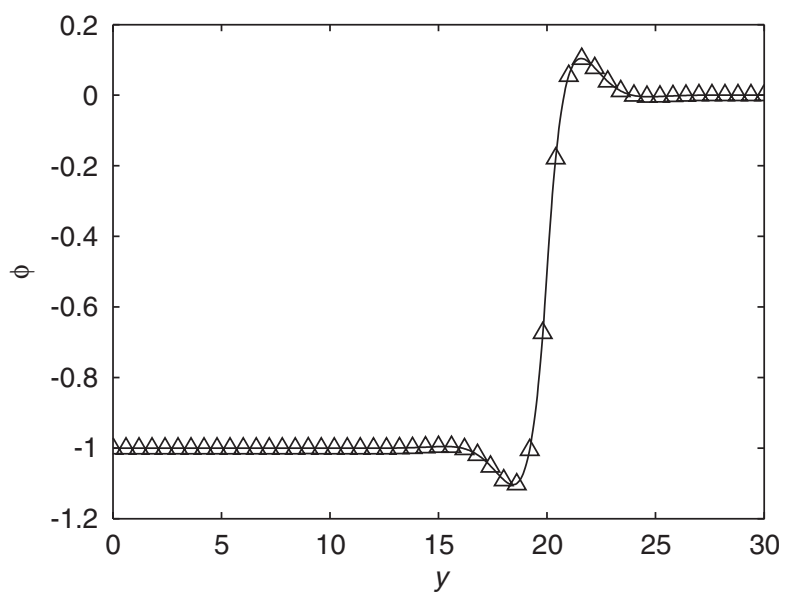

Fig. 3. Analytic (solid) and numerical $(\triangle)$ solution at time $t=20$ for the case $\Delta \rightarrow 0, k_{x}=k_{z}=\psi=0$ and $\omega=\sigma_{0}=c=1$. (This corresponds to $M \rightarrow \infty, G=0, \Omega=1$ using the definitions in Section 2.2.)

which for fixed $y$ decays over time $t \rightarrow \infty$ to the constant given by the second term on the right-hand side. An example for $\psi=0$ and $\omega=\sigma_{0}=c=1$, starting the calculation at $t=-10$ and ending at $t=20$, is shown in Fig. 3, where the real part of $\phi$ is plotted. The analytical solution given by Eqs. (8) and (9) has also been used to validate a numerical solution (shown on the figure using symbols) based on second-order central differencing in space and time. In the numerical solution the surface forcing is given by the real part of Eq. (2).

\subsection{Numerical solution for arbitrary growth rate and wavenumber}

Solutions for arbitrary $k_{x}$, holding $k_{z}=0$, are obtained numerically. To simplify the presentation of results, we identify three dimensionless parameters. A Mach number (based on the phase speed of the unmodulated wave) is defined as

$$
M=\frac{\omega}{k_{x} c},
$$

a saturation parameter by

$$
G=\sigma_{0} \Delta
$$

and a normalised frequency by

$$
\Omega=\frac{\omega}{\sigma_{0}} .
$$

It may be noted that any spanwise wavenumber is contained in the solutions that follow, by substituting $k=\sqrt{k_{x}^{2}+k_{z}^{2}}$ for $k_{x}$. The Mach number in Eq. (10) is the ratio of the phase speed along the boundary to the sound speed in the fluid; $G$ is twice the growth exponent during the saturation process $(-\Delta<t<0)$; and $\Omega$ is the ratio of the frequency to the initial growth rate. Fig. 4 shows a result at $t=20$ for $M=0.5, G=1$ and $\Omega=1$, illustrating the generation of sound from a subsonic mode. The calculation was started at $t=-10$; however the results are insensitive to start time so long as it is a sufficiently large negative number.

To measure the relative acoustic output of the solution, we introduce a norm $N$, defined by

$$
N=\frac{-\int_{-\infty}^{+\infty}(\partial \phi / \partial y)_{0}(\partial \phi / \partial t)_{0} \mathrm{~d} t}{c \int_{-\infty}^{+\infty}(\partial \phi / \partial y)_{0}^{2} \mathrm{~d} t},
$$

where the subscript 0 implies $y=0$. Introducing a factor $\rho$ (the unperturbed density) in both numerator and denominator makes the numerator equal to the integral of pressure times normal velocity, i.e. the radiated acoustic energy per unit area of boundary, while the denominator is a normalising factor equal to the energy 


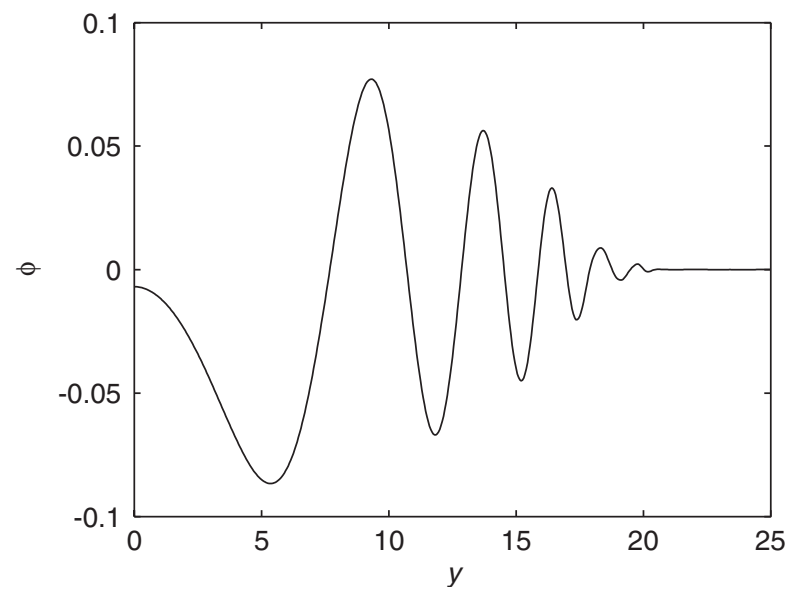

Fig. 4. Numerical solution at $t=20$ for $M=0.5, G=1, \Omega=1$ and $\psi=0$.

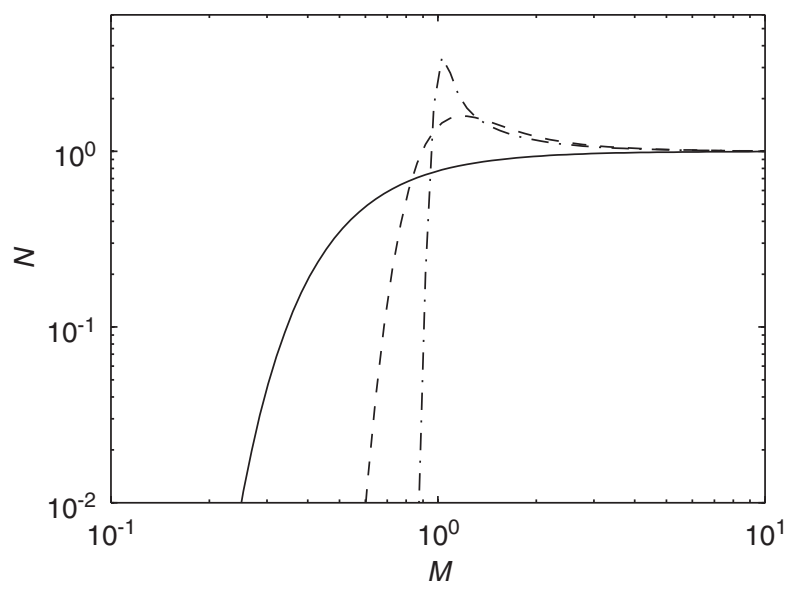

Fig. 5. Variation of the norm $N$, illustrating cutoff with decreasing Mach number $M$ for various frequencies $\Omega ; G=10, \psi=0 ;-$, $\Omega=0.2 ;---, \Omega=1 ;-\cdot-, \Omega=5$.

radiated for zero-wavenumber forcing $\left(k_{x}=0\right)$. In Appendix $\mathrm{A}$ it is shown that this norm is invariant to the choice of integration surface. Its value is in reducing each simulation output, of a form typified by Fig. 3, to a single numerical value that measures the radiation efficiency of the forcing.

Simulations have been carried out to map out the cutoff dependence on $M, G$ and $\Omega$, as illustrated in Figs. 5-7. Fig. 5 shows the variation of the norm $N$ with Mach number $M$ for several $\Omega$, computed for $G=10$. For large $\Omega$ (i.e. temporal growth rate $\sigma_{0}$ is relatively unimportant) we recover the standard result [4] that radiation is only significant for $M>1$. For lower frequencies $\Omega$ there is still significant radiation for $M<1$, and as the frequency is further reduced one needs to go to quite low subsonic Mach numbers before the radiated energy is significantly cut off.

Fig. 6 shows the effect of frequency $\Omega$ and phase $\psi$ on radiation from subsonic modes $(M=0.5)$, again for $G=10$. It can be seen that only low frequencies radiate significant energy and that the effect of phase disappears at low and high frequencies $(N \rightarrow 1$ and $N \rightarrow 0$, respectively). In the intermediate region there is an effect of the phase $\psi$, three values for which $(\psi=0, \psi=\pi / 4$ and $\psi=\pi / 2)$ are shown in the figure. Fig. 7 shows the effect of varying both $G$ and $\Omega$ for the case $(\psi=0, M=0.5) ; N$ is plotted against the combined saturation and frequency parameter $G \Omega^{2}$ for various $\Omega$. A collapse of the curves for different frequencies is obtained for high values of $G \Omega^{2}$. We observe that there is a clear cutoff phenomenon for $G \Omega^{2} \gg 1$, 


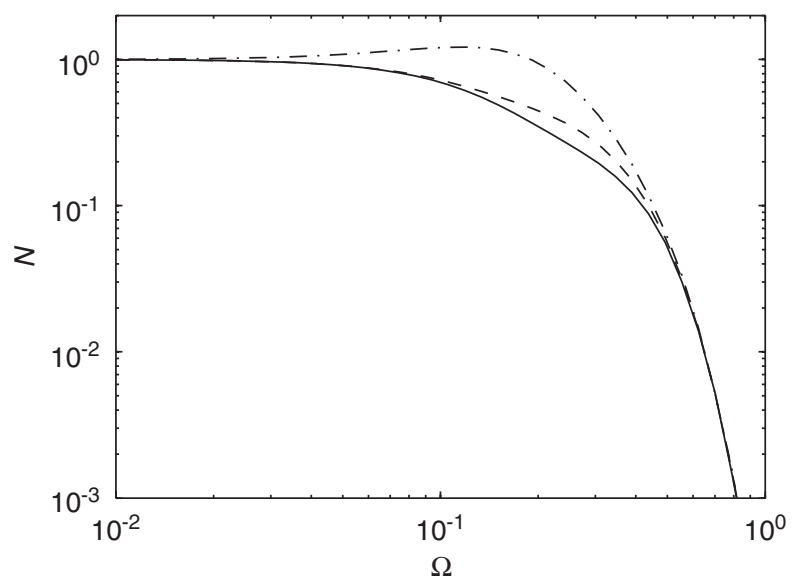

Fig. 6. Variation of the norm $N$, illustrating cutoff with frequency $\Omega$ for various phases $\psi ; G=10, M=0.5 ;-, \psi=0 ;---, \psi=\pi / 4$; $-\cdot, \psi=\pi / 2$.

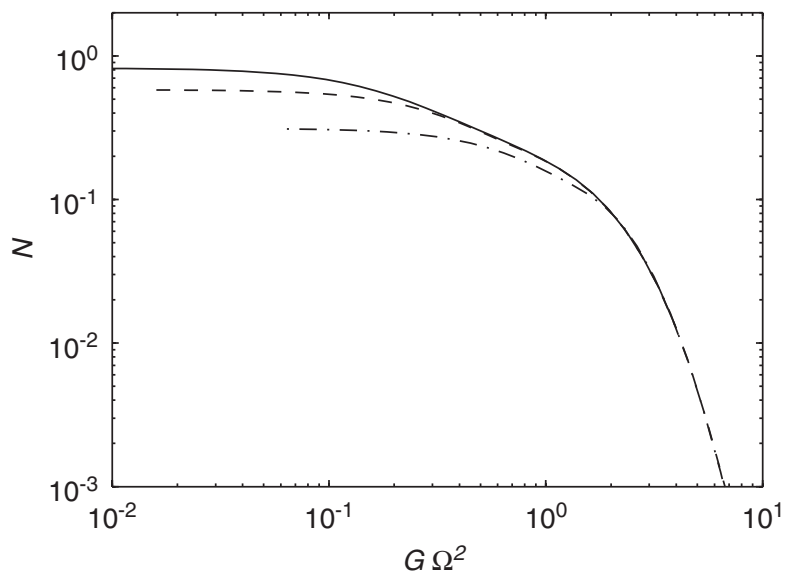

Fig. 7. Variation of the norm $N$, illustrating cutoff with the combined saturation parameter $G \Omega^{2}$ for various frequencies $\Omega$; $M=0.5$; $\psi=0 ; \_, \Omega=0.2 ;---, \Omega=0.4 ;-\cdot-, \Omega=0.8$.

corresponding to the case where the initial growth is slow $\left(\sigma_{0} \ll \omega^{2} \Delta\right)$ or saturation occurs over a long time scale. On the other hand, when $G \Omega^{2}<1$, corresponding to a sufficiently rapid initial growth with $\sigma_{0}>\omega^{2} \Delta$, the cutoff (even at $M=0.5$ ) is relatively weak, with a radiation efficiency of at least $15 \%$ for all the $\Omega$ considered.

\section{Conclusions}

Analytical and numerical results from a model problem in acoustics have been used to illustrate the sound radiated from subsonic travelling-wave disturbances in the form of a growing and decaying wave packet, such as may occur in transitional and turbulent shear flows. The convective growth and decay is replaced in the model by the temporal growth and decay of a spatially-sinusoidal travelling wave. It has been shown how sound radiation is progressively cut off as the phase speed is reduced relative to the ambient sound speed, particularly for high frequencies and slow mode saturation. However, when the initial growth rate is sufficiently rapid, or the saturation phase is of sufficiently short duration, the cutoff of subsonic waves tends to disappear and the radiation efficiency approaches unity regardless of Mach number.

In the subsonic jet noise problem, a possible mechanism of sound radiation is the non-linear (quadratic in the first instance) interaction between instability waves with similar streamwise wavenumbers $k_{1}$ and $k_{1}+\delta k$. On a near-parallel base flow these grow exponentially (with nearly constant exponent), starting from a very 
small amplitude. As they reach high amplitude the associated Reynolds stresses lead to an increase in shear layer thickness and hence saturation, the same wavelengths now being stable on the changed base flow. Among the wavenumbers generated by such an interaction is the low wavenumber $\delta k$ (and hence low frequency, for a given group velocity) that satisfies all the conditions for radiation given here, even when the convective Mach number arising from the interaction is subsonic.

\section{Appendix A. Invariance of the norm $N$}

Using the chain rule and substituting the wave equation (1) we can write a conservation law in local form as

$$
\frac{\partial}{\partial y}\left(\frac{\partial \phi}{\partial y} \frac{\partial \phi}{\partial t}\right)=\frac{\partial E}{\partial t}
$$

with

$$
E=\frac{1}{2 c^{2}}\left(\frac{\partial \phi}{\partial t}\right)^{2}+\frac{1}{2} k^{2} \phi^{2}+\frac{1}{2}\left(\frac{\partial \phi}{\partial y}\right)^{2},
$$

where $k^{2}=k_{x}^{2}+k_{z}^{2}$. Integration of Eq. (A.1) from $y=0$ to $y=y_{1}$, and from $t=-\infty$ to $t=\infty$, gives

$$
\left[\int_{-\infty}^{\infty} \frac{\partial \phi}{\partial y} \frac{\partial \phi}{\partial t} \mathrm{~d} t\right]_{y=0}^{y=y_{1}}=\left[\int_{0}^{y_{1}} E \mathrm{~d} y\right]_{t=-\infty}^{t=\infty} .
$$

The right-hand side is zero, since $E$ is zero for all $y$ at both $t=-\infty$ and $t=\infty$. It follows that

$$
\int_{-\infty}^{\infty}\left(\frac{\partial \phi}{\partial y}\right)_{1}\left(\frac{\partial \phi}{\partial t}\right)_{1} \mathrm{~d} t=\int_{-\infty}^{\infty}\left(\frac{\partial \phi}{\partial y}\right)_{0}\left(\frac{\partial \phi}{\partial t}\right)_{0} \mathrm{~d} t
$$

and the numerator of the norm $N$ defined in Eq. (13) is invariant with respect to integration surface: it will have the same value whether calculated at the wall or in the far field $k y \gg 1$.

\section{References}

[1] C. Tam, Supersonic jet noise, Annual Review of Fluid Mechanics 27 (1996) 17-43.

[2] M.J. Lighthill, On sound generated aerodynamically, Proceedings of the Royal Society of London, Series A, vol. 211, 1952 , pp. $564-587$.

[3] H. Liepmann, A. Roshko, Elements of Gasdynamics, Wiley, New York, 1957 (see Chapter 8, Sections 8.5 and 8.6).

[4] L. Cremer, M. Heckl, Structure-Borne Sound (second edition translated and revised by E.E. Ungar), Springer, Berlin, 1988 (see Chapter VI, Sections $4 \mathrm{~b}$ and $6 \mathrm{~b})$.

[5] D.G. Crighton, P. Huerre, Shear-layer pressure fluctuations and superdirective acoustic sources, Journal of Fluid Mechanics 220 (1990) $355-368$. 